\title{
Estratégia de Promoção à Saúde: Programa Academia da Cidade de Belo Horizonte
}

\section{Health Promotion Strategy: Academia da Cidade Program of Belo Horizonte}

Aline Cristine Souza Lopes',Aline Dayrell Ferreira ${ }^{2}$,Raquel de Deus Mendonça' ${ }^{1}$, Maria Angélica Salles Dias³,

Rony Carlos Las Casas Rodrigue 3 , Luana Caroline Santos

\section{Resumo}

O artigo tem o objetivo de descrever o histórico, o propósito e as ações de promoção saúde realizadas no polo piloto do Programa Academia da Cidade (PAC) de Belo Horizonte-MG. O PAC foi implantado em 2005 com a finalidade de promover modos saudáveis mediante intervenções interdisciplinares, prioritariamente coletivas, que ampliassem a autonomia dos sujeitos, respeitando a diversidade cultural da comunidade. O primeiro polo ofertava exercícios físicos e aconselhamento nutricional integrado às Unidades Básicas de Saúde. Atendia cerca de 400 pessoas que apresentavam baixo nível econômico, alimentação não saudável, excesso de peso e comorbidades. As ações propiciaram a prática regular de exercícios físicos, adoção de hábitos alimentares saudáveis e redução do peso. O PAC contribui para a promoção da saúde com equidade e justiça social.

\section{Palavras-chave}

Serviços de saúde; Intervenções comunitárias; Promoção da saúde; Sistema Único de Saúde.

\begin{abstract}
The article aim to describe the history, purpose and health promotion action developed in the pilot Programa Academia da Cidade (PAC) in Belo Horizonte-MG. The PAC was implemented in 2005 to promote healthy ways of life through interdisciplinary interventions, mainly collective, which amplify the autonomy of individuals and to consider health demands of community, respecting cultural diversity. The first unit offering regular physical exercise and nutritional counseling integrated into basic bealth unit. The unit attends approximately 400 people who low income, unhealthy diet, excess of weight and comorbidities. The actions provided regular physical exercise, adopting healthier eating habits and weight reduction. The $P A C$ contributes to bealth promotion with equity and social justice.
\end{abstract}

\section{Keywords}

Health Services; Community-Based Interventions; Health Promotion; Unified Health System.

\section{Introdução}

A promoção da saúde emerge como demanda devido às transformações sociais, econômicas e de saúde vivenciadas pela sociedade ${ }^{1}$. No Brasil, políticas e programas têm sido propostos. Destaca-se o Programa Academia da Cidade (PAC), implantado a partir de 2002 em algumas capitais brasileiras ${ }^{2}$, sendo em 2011 instituído nacionalmente como Programa Academia da Saúde ${ }^{3}$.

Atualmente, o Programa é um ponto de atenção do Sistema Único de Saúde (SUS) caracterizado por espaços físicos, denominados polos, com infraestrutura, equipamentos e recursos humanos qualificados para a orientação de práticas corporais, atividade fí-

1 Universidade Federal de Minas Gerais, Departamento de Nutrição, Belo Horizonte, Minas Gerais, Brasil.

2 Secretaria Municipal de Saúde de Contagem; Belo Horizonte, Minas Gerais, Brasil. 3 Secretaria Municipal de Saúde de Belo Horizonte, Belo Horizonte, Minas Gerais, Brasil. sica e lazer, modos saudáveis de vida e alimentação saudável ${ }^{3}$.

Este trabalho objetivou descrever o histórico, os propósitos e as ações realizadas no polo piloto do Programa Academia da Cidade em Belo Horizonte-MG.

\section{Programa Academia da Cidade de Belo Horizonte - MG}

A criação do PAC em Belo Horizonte foi oriunda da necessidade de estratégias intersetoriais que abarcassem as necessidades em saúde da população, com destaque para a melhor estruturação da promoção da saúde e do cuidado aos usuários com doenças crônicas não transmissíveis (DCNT).

Os gestores, para implantarem o PAC, visitaram experiências de outras cidades. E, em parceria com o Ministério da Saúde, o município ingressou em uma 
rede de colaboração com Centro de Controle e Prevenção de Doenças dos Estados Unidos (CDC), Projeto GUIA (Guia Útil de Intervenções para Atividade no Brasil e América Latina), universidades brasileiras e outras prefeituras (Recife-PE, Vitória-ES e Aracaju-SE) com vistas à sistematização do Programa ${ }^{2}$. Adicionalmente, no ano de 2005, a Secretaria de Vigilância em Saúde fomentou diversas ações para promoção da saúde, sendo um deles o repasse de recursos para projetos de atividade física no SUS 4 .

Concomitantemente, foi criado no município o Grupo Intersetorial de Promoção à Saúde para o planejamento do PAC. Este grupo era formado por profissionais das Secretarias Municipais de Saúde, Segurança Alimentar e Nutricional, Esportes e Educação, e da Universidade Federal de Minas Gerais ${ }^{5}$.

Nos encontros, o Grupo definiu os objetivos do PAC, o processo de ingresso e acompanhamento dos usuários e quais atividades que seriam realizadas. Após discussões com a comunidade e equipamentos sociais, o polo piloto foi inaugurado em 2005.

\section{Objetivo e funcionamento do PAC}

O principal objetivo do PAC era promover modos saudáveis (atividade física e alimentação saudável) mediante intervenções interdisciplinares, prioritariamente coletivas, que ampliassem a autonomia dos sujeitos.

As ações eram realizadas, no polo piloto, por duas equipes. Uma de educação física que consistia de um profissional e três estagiários vinculados à Prefeitura Municipal e a outra de nutrição, formada por duas nutricionistas e duas alunas da graduação em Nutrição, orientadas por um professor da Universidade Federal de Minas Gerais (UFMG).

Em Belo Horizonte, atualmente, há 63 polos do PAC os quais são geridos por educadores físicos que, a partir de 2008, passaram a integrar o Núcleo de Apoio à Saúde da Família (NASF), pois no período de planejamento e implantação do polo piloto ainda não existia o NASF no País. Em 2009, os profissionais do NASF (nutricionista, psicólogo, fisioterapeuta e fonoaudiólogo) começaram a apoiar o polo e, a partir, de 2012, as ações de alimentação passaram a ser supervisionadas pelo nutricionista do NASF e conduzidas por estudantes de nutrição, orientados por um docente da UFMG.

Na Figura 1 é apresentado o modelo lógico que descreve os insumos, ações, produtos e resultados.

\section{O Ingresso do Usuário no PAC}

No PAC, o acesso é universal, todos os interessados podem participar das ações ofertadas, seja por demanda espontânea ou encaminhamento dos serviços de saúde, sobretudo das Unidades Básicas de Saúde (UBS). Devido à alta demanda e o limite da capacidade de atendimento, os interessados realizavam inscrição prévia, em datas e horários previamente divulgados (Figura 2).

O usuário, depois de inscrito, participava de avaliação física e nutricional, e reavaliação anual. $\mathrm{Na}$ avaliação física o usuário era classificado segundo o risco para realização de exercícios físicos, sendo os indivíduos com baixo e moderado risco, considerados aptos. Enquanto que, aqueles com alto risco eram classificados como inaptos e encaminhados para a UBS para a propedêutica necessária, podendo ser reencaminhados por laudo médico. A avaliação da aptidão física era realizada pelo educador físico com anamnese específica. Posteriormente, a equipe de nutrição da UFMG realizava avaliação nutricional. Todos os dados eram registrados em computador para posterior reavaliação (Figura 2). 


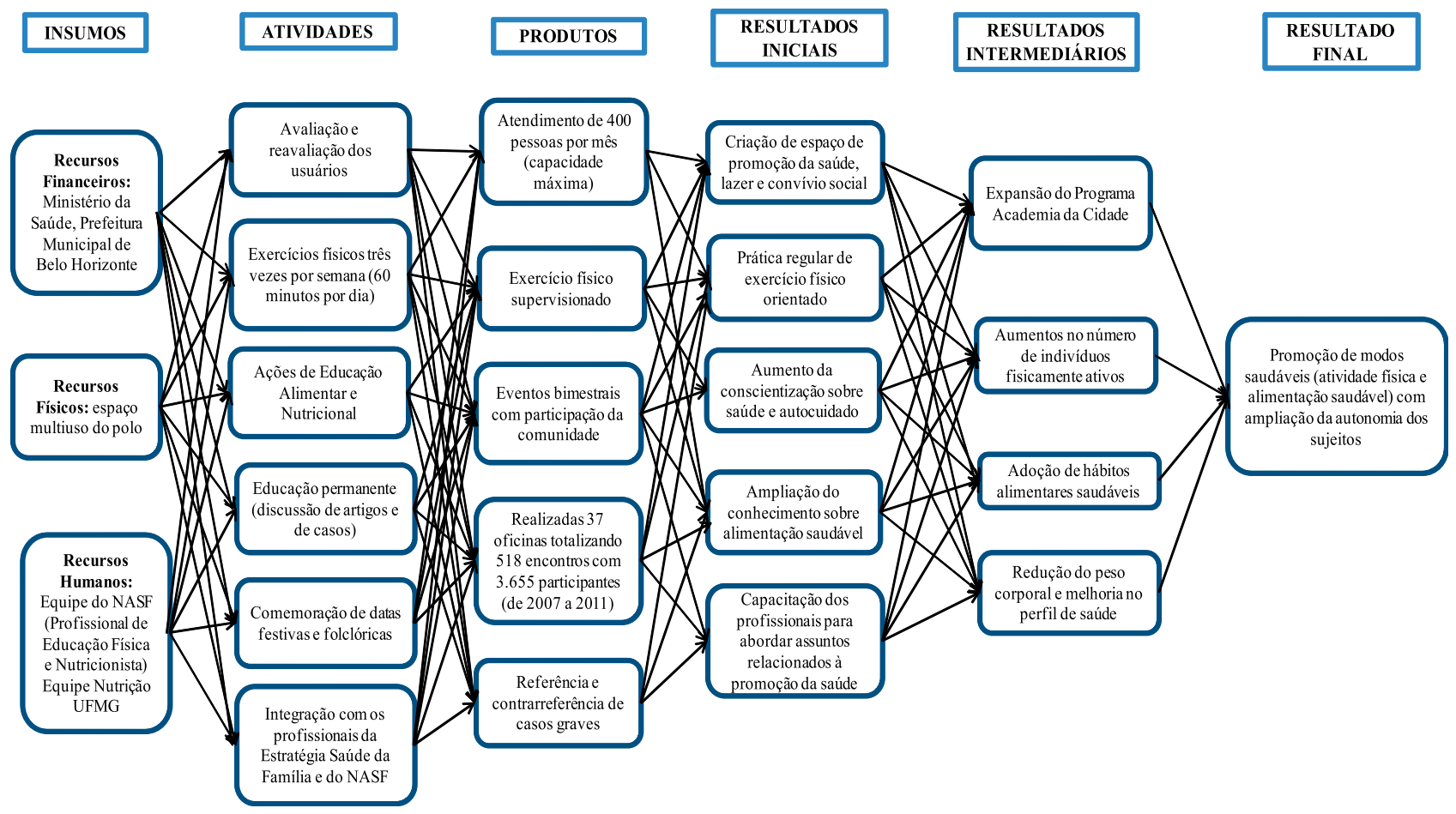

FIGURA 1 - Modelo Lógico.

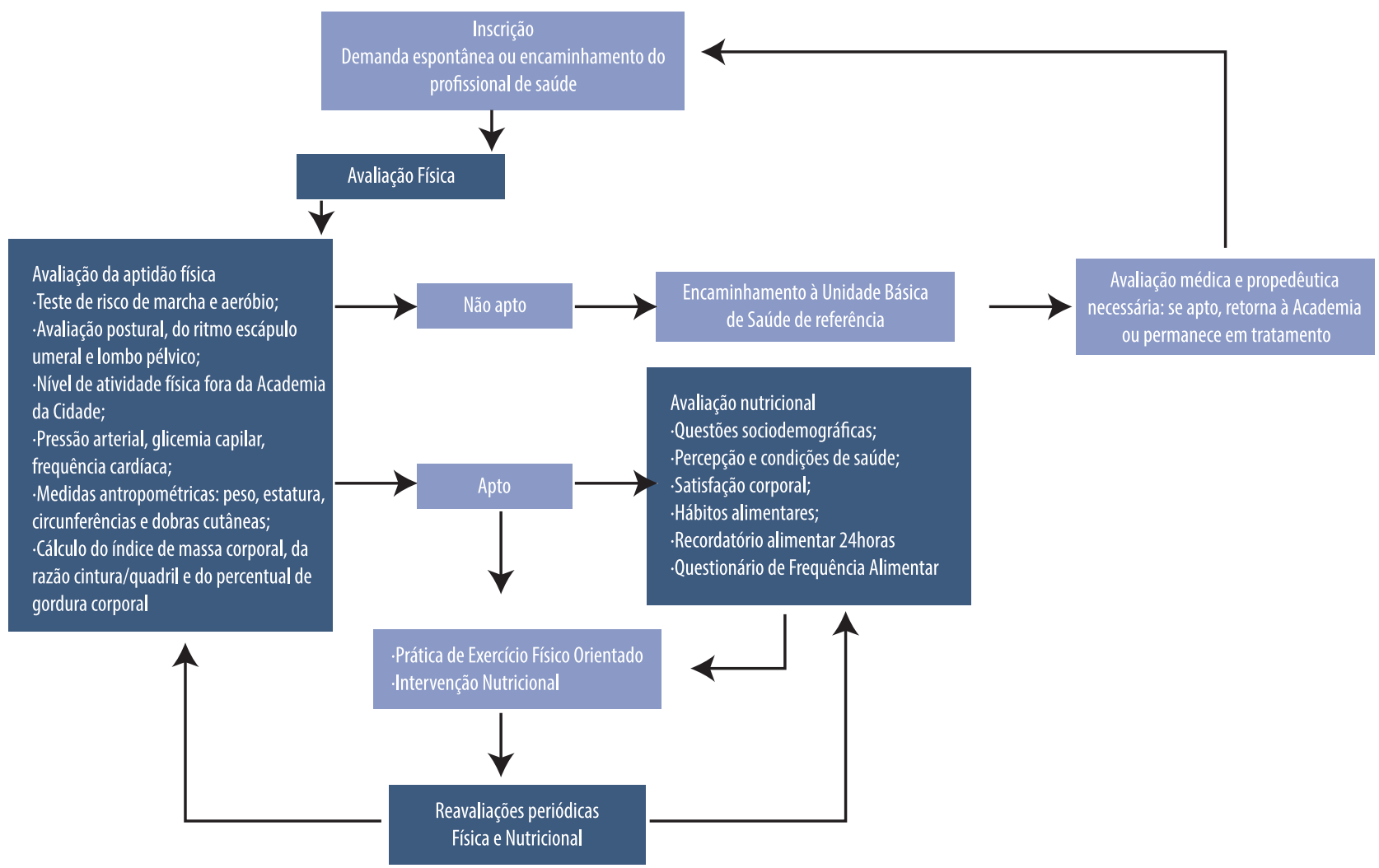

FIGURA 2 - Fluxo esquemático do atendimento no Programa Academia da Cidade. Belo Horizonte.

Para favorecer o cuidado integral à saúde foi estabelecido um fluxo de referência e contrarreferência com a UBS. Além de participar das ações do polo, os casos graves de indivíduos com obesidade e DCNT desestabilizadas, especialmente dia- 
betes e hipertensão arterial, eram encaminhados para acompanhamento nutricional individual, em parceria com universidade e apoio da Estratégia de Saúde da Família (ESF).

Resultados das avaliações dos usuários que ingressaram no PAC, de fevereiro a setembro de 2007 , apontaram elevadas prevalências de excesso de peso (77,7\%), hipertensão arterial (49,5\%) e diabetes mellitus (15,7\%), além de elevados níveis de sedentarismo e alimentação não saudável ${ }^{6}$.

\section{Ações do PAC: Exercício Físico e Alimentação Saudável}

Os exercícios físicos eram, realizados três vezes por semana com 60 minutos de duração diária, geralmente distribuídos em 30 minutos de atividades aeróbias e 30 minutos com exercícios que exploram as demais capacidades físicas relacionadas à saúde. Os interessados podiam escolher o horário para praticar exercício físico de 08 as 12 horas da manhã. Diariamente era registrada a frequência do usuário e mensalmente contabilizadas as desistências, sendo estes acessados por telefone para verificar os motivos do abandono e estimular o retorno ao serviço. Os usuários também eram orientados para a prática de exercício físico em outros espaços públicos da cidade, como praças, parques e avenidas.

As ações de alimentação constaram de grupos interdisciplinares, mensais e abertos, com objetivo de aumentar a autonomia para escolhas saudáveis aplicáveis à realidade. As atividades eram realizadas no formato de oficinas, com duração de 60 minutos, realizadas durante uma semana em dias e horários distintos, para oportunizar a participação dos usuários. Ao total, as oficinas eram reaplicadas 14 vezes por semana. Os temas eram selecionados de acordo com a avaliação inicial, dúvidas e questionamentos dos usuários. Na técnica de oficinas os participantes desenvolvem uma determinada tarefa e seguem as seguintes etapas: aquecimento, reflexão individual, reflexão grupal e conclusão 7 . Por exemplo, em uma oficina realizada para discutir o custo da alimentação saudável, inicialmente, os usuários eram estimulados a expor suas opiniões sobre o preço dos alimentos saudáveis e a interferência deste aspecto na saúde. A seguir, eram convidados para simular uma compra de alimentos saudáveis dentro de um valor estipulado (um espaço era preparado com materiais necessários - cesta de compras, réplicas de alimento e notas falsas de dinheiro). Após a realização da tarefa, uma discussão em grupo era coordenada pelo profissional, abordando as barreiras vivenciadas diariamente para aquisição de alimentos saudáveis e possíveis soluções, finalizando com uma conclusão construída conjuntamente pelos participantes.

Adicionalmente, ações com enfoque sociocultural, como comemoração das datas festivas e folclóricas, eram desenvolvidas. Atividades de educação permanente eram desenvolvidas rotineiramente entre os profissionais de educação física e de nutrição. Elas ocorriam uma vez por semana, com duração de 30 a 40 minutos, abordando temas de acordo com as necessidades dos profissionais e estagiários. Eram discutidos artigos científicos, casos de usuários com dificuldades para adesão ou necessidades especiais, bem como problemas identificados no cotidiano do serviço. Estas discussões tinham a finalidade de melhorar as ações realizadas no PAC e planejar estratégias para casos específicos, como o referenciamento de usuários para outros pontos de atenção da rede.

\section{Impacto do PAC}

Como primeiro reflexo positivo da implantação do PAC no município destaca-se a sua expansão. Atualmente, estão em funcionamento 63 polos, em três turnos 
(manhã, tarde e noite), que atendem aproximadamente 24.000 usuários, com vistas a ampliar nos próximos anos, sobretudo no período noturno, maior demanda da população.

No primeiro ano de implantação, o polo piloto atingiu a capacidade máxima de atendimento, 400 usuários. De 2007-2011 foram realizadas 37 oficinas relativas à cidadania, saúde e nutrição, totalizando 518 encontros com 3.655 participantes. A avaliação dos usuários mais graves, encaminhados para acompanhamento nutricional individual na UBS, de agosto de 2008 a maio de 2009, revelou melhorias na alimentação, redução da massa corporal e obesidade abdominal ${ }^{8}$. A partir do monitoramento das avaliações foi possível observar que a associação entre exercício físico e intervenção nutricional repercutiu em hábitos alimentares saudáveis e redução ponderal ${ }^{9}$.

Os efeitos positivos verificados corroboram políticas internacionais e nacionais que visam o controle das DCNT, como o Plano de Ações Estratégicas para o Enfrentamento das Doenças Crônicas não Transmissíveis no Brasil ${ }^{10}$. E, atualmente, o PAC integra o Programa Academia da Saúde do Ministério da Saúde. Ademais, os usuários do polo relataram satisfação com o serviço, descrevendo-o como espaço de promoção da saúde, lazer e convívio social, como pode ser verificado nas falas:

Pra mim sentir bem!Pra en ter uma motivação! Assim pra viver!? Por que eu tava muito triste, eu tava com início de depressão e agora melhorou, e o colesterol era altíssimo e melhorou, ficou tudo normal, pressão!

Ahbh.... É um incentivo!...De alimentação, de como é necessário fazer um exercício físico e uma caminhada! Um alongamento. E agora eu não fico sem, não consigo. Tem vez que dá desânimo de vim a pé aí eu falo: "não, eu preciso, eu tenho aqui." e fora as amizades!? As amizades assim, conversa com as pessoas?! A gente sai da rotina.

A oportunidade de convívio social ofertada pelo PAC tem sido destaque em suas ações por possibilitar maior integração entre a comunidade e os serviços, potencializando os efeitos da promoção da saúde ${ }^{11}$. E, é importante frisar a alta carga de doenças da população atendida ${ }^{12}$ que demonstra a importância da rede de atenção à saúde (PAC/UBS/ESF/NASF).

O PAC demonstra ser um serviço ideal para a promoção da saúde, entretanto, desafios ainda são vivenciados, com a infraestrutura física e recursos humanos. $\mathrm{O}$ espaço coberto reduzido prejudica as ações coletivas simultâneas devido a condições climáticas.

Os profissionais do NASF atendem um território com muitas demandas por sua vulnerabilidade e composição, e, devido à alta carga de trabalho, possuem pouca disponibilidade para planejar, construir e desenvolver ações coletivas, além da escassez de material didático para apoiar estas atividades. A falta de comunicação e o trabalho interdisciplinar entre o polo, profissionais da ESF e NASF é um limitante.

\section{Considerações finais}

A oferta de prática regular de exercícios físicos e acompanhamento nutricional para a comunidade mediante a prática interdisciplinar e em rede propicia o enfrentamento das DCNT e a promoção de modos saudáveis de viver. Ademais, a socialização oportunizada por estratégias coletivas, como o PAC, favorece a integração entre os usuários e profissionais, com reforço das ações desenvolvidas. Todas estas características evidenciam a capacidade do PAC em oportunizar a promoção e a recuperação da saúde no cotidiano de indivíduos e comunidades. 


\section{Auxílio Financeiro}

Fundação de Amparo à Pesquisa do Estado de Minas Gerais (FAPEMIG), processo número CDS APQ-0376-4.08/07. Secretaria Municipal de Saúde de Belo Horizonte - Minas Gerais.

\section{Contribuiç̧ão}

ACSL: concepção e desenho do trabalho, e redação do manuscrito. ADF: concepção e desenho do trabalho, e revisão do manuscrito. RDM: coleta de dados e redação do manuscrito. MASD: concepção e revisão do manuscrito. RCLCR: concepção e desenho do trabalho, e revisão do manuscrito. LCS: concepção e desenho do trabalho, e revisão do manuscrito. Todos os autores aprovaram a versão final do artigo.

\section{REFERÊNCIAS}

1. Brasil. Ministério da Saúde. Secretaria de Vigilância da Saúde. Secretaria de Atenção à Saúde. Política Nacional de Promoção da Saúde: PNPS: revisão da Portaria MS/GM no 687, de 30 de março de 2006. Brasília: Ministério de Saúde, 2014.

2. Brasil. Ministério da Saúde. Saúde Brasil 2010: Uma análise da situação de saúde e de evidências selecionadas de impacto de ações de vigilância em Saúde. Brasília: Ministério da Saúde, 2010.

3. Brasil. Ministério da Saúde. Portaria $n^{\circ} 2.681$, de 7 de novembro de 2013. Redefine o Programa Academia da Saúde no âmbito do Sistema Único de Saúde. 2013. [citado 2014 jan 15]. Disponível em:http:/bvsms.saude.gov.br/bvs/saudelegis/gm/2013/ prt2681_07_11_2013.html

4. Malta DC, Castro AM, Cruz DKA, Gosh CS. A promoção da saúde e da atividade física no sistema único de saúde. Rev Bras Ativ Fís Saúde. 2008; 13 (1):24-7.

5. Dias MAS, Lopes ACS, Bicalho K, Mourão M, Alves MN, Evangelista PA, et al. Promoção à saúde e articulação intersetorial. In: Magalhães Júnior HM (Org). Desafios e inovações na gestão do SUS em Belo Horizonte: a experiência de 2003 a 2008. Belo Horizonte: Mazza Edições; 2010. p. 63-98.

6. Lopes ACS, Reyes ANL, Cesar CC, Menezes MC, Santos LC. Fatores associados ao excesso de peso entre mulheres. Esc Anna Nery. 2012; 16 (3): 451-8.

7. Afonso, L. Oficinas em dinâmica de grupo. In: Afonso, L. Oficinas em dinâmica de grupo: um método de intervenção psicossocial. Belo Horizonte: Edições do Campo Social, 2002. p.11-59.

8. Ferreira NL, Mingoti SA, Jaime PC, Lopes ACS. Effectiveness of nutritional intervention in overweight women in Primary Health Care. Rev Nutr. 2014; 27 (6): 677-87.

9. Mendonça RM, Lopes ACS. The effects of health interventions on dietary habits and physical measurements. Rev Esc Enferm USP. 2012; 46 (3):573-9.

10. Brasil. Ministério da Saúde. Secretaria de Vigilância em Saúde. Departamento de Análise de Situação de Saúde. Plano de ações estratégicas para o enfrentamento das doenças crônicas não transmissíveis (DCNT) no Brasil 2011-2022. Brasília: Ministério da Saúde, 2011.

11. Silva KL, Sena RR, Matos JA, Lima KMS, Silva PM. Acesso e utilização da Academia da Cidade de Belo Horizonte: perspectiva de usuários e monitores. Rev Bras Ativ Fís Saúde. 2014; 19 (6): 700-10.

12. Costa BVL, Mendonça RD, Santos LC, Peixoto SV, Alves M, Lopes ACS. Academia da Cidade: um serviço de promoção da saúde na rede assistencial do Sistema Único de Saúde. Cien Saude Colet. 2013; 18 (1): 95-102.

ENDEREÇO PARA

CORRESPONDÊNCIA

ALINE CRISTINE SOUZA LOPES

alinelopesenf@gmail.com
Departamento de Nutrição da Escola de Enfermagem.

Avenida Alfredo Balena, 190, sala 316,

Santa Efigênia. CEP: 31.660-000, Belo

Horizonte, Minas Gerais. Brasil.

Telefone: 5531 3409-9179; Fax.: 5531

3409-8028 - e-mail: 